\title{
Neurolingüística: de cómo el cerebro bilingüe aprende palabras
}

\author{
Haydee Carrasco Ortiz y Flora Beatriz Hernández Carrillo
}

\begin{abstract}
Resumen
Aprender otras lenguas nos da la oportunidad de asombrarnos y cuestionarnos sobre la manera cómo nuestro cerebro incorpora nuevas palabras a su repertorio léxico. De manera general, la neurolingüística busca revelar aquellas operaciones mentales que el cerebro realiza durante el aprendizaje y el procesamiento de los estímulos lingüísticos. Una de las cuestiones fundamentales en el estudio del bilingüismo, bajo el enfoque neurolingüístico, es determinar de qué forma el cerebro bilingüe almacena y recupera la información relacionada con las palabras en cada una de sus lenguas. En específico, ¿cómo activamos el significado de una palabra en una y otra lengua? o ¿cómo el cerebro toma una palabra y la divide (o no) en sus distintas unidades ortográficas? En este artículo haremos una revisión de los principales hallazgos acerca de cómo el cerebro bilingüe representa y organiza las palabras en cada una de sus lenguas. Finalmente, se discuten algunas implicaciones para el ámbito educativo y futuros estudios que busquen contribuir al acercamiento entre las ciencias del aprendizaje y las neurociencias.
\end{abstract}

Palabras clave: neurolingüística, enseñanza de segunda lengua, neuroimagen cerebral, bilingüismo.

\section{NEUROLINGUISTICS: HOW A BILINGUAL BRAIN LEARNS WORDS}

\begin{abstract}
Second language word learning is an extremely complex and fascinating mental process that can help us to understand how our brain incorporates new words into its lexicon. In general, neurolinguistics seeks to reveal the mental processes associated with learning and processing of a linguistic stimulus. One of the fundamental issues addressed in bilingual studies, under the neurolinguistic approach, is to determine how the bilingual brain stores and retrieves information related to words in each of its two languages. Specifically, how do we activate word meaning in both languages? Or how does our brain take a word and divide it (or not) into its different orthographic components? In this article we review the main findings regarding these questions as well as evidence of how the bilingual brain represents and organizes words in each of its two languages. Finally, we discuss some implications for the educational field.
\end{abstract}

Keywords: neurolinguistics, second language teaching, cerebral neuroimaging, bilingualism.

Dol: http://doi.org/10.22201/codeic.16076079e.2020.v21n3.a6 
"Neurolingüística: de cómo el cerebro bilingüe aprende palabras" Haydee Carrasco Ortiz y Flora Beatriz Hernández Carrillo

\section{Haydee Carrasco Ortiz}

haydee.carrasco@uaq.edu.mx https://orcid.org/0000-0002-2776-5965

Docente-investigadora en la Facultad de Lenguas y Letras de la Universidad Autónoma de Querétaro. Realizó estudios de licenciatura en Psicología en la unam. Obtuvo su maestría y doctorado en Psicología Cognitiva en la Universidad Aix-Marsella, Francia y realizó estudios de postdoctorado en la Universidad de Tufts y en el Departamento de Brain and Cognitive Sciences en mit (Instituto Tecnológico de Massachusetts) en Estados Unidos. Sus áreas de interés son la neurolingüística y el bilingüismo. Específicamente, se interesa en los mecanismos cerebrales y cognitivos involucrados en la comprensión de lenguaje y en el reconocimiento visual de palabras tanto de la lengua materna como de la segunda lengua.

\section{Flora Beatriz Hernández Carrillo}

florahc@unam.mx https://orcid.org/0000-0002-3858-6161

Doctorante en psicología educativa y del desarrollo (Facultad de Psicología, unam). Investigadora del proyecto "Aprendiendo Juntos" y co-autora del capítulo Dialogical Interactions among peers in collaborative writing contexts. Ha trabajado en el sector educativo y empresarial desde hace 13 años. Investigadora en tópicos de psicopedagogía, desarrollo profesional e interacción sectores educativo-productivo. Actualmente, Coordinadora de Investigación Traslacional en Educación de la CODEIC, UNAM. 


\section{Introducción}

Aprender vocabulario es esencial para lograr comprender una segunda lengua (L2). Muchas veces, aprender una palabra en otra lengua implica asignar un nuevo significado a una forma ortográfica ya conocida en nuestra lengua materna (L1). Cuántos de nosotros no nos sorprendimos cuando descubrimos que la palabra en inglés diversion, escrita en un letrero junto con una flecha, no señala la ruta a la diversión, sino simplemente anuncia una desviación del camino más adelante. Aprender una nueva palabra requiere asociar una serie de letras o unidades ortográficas a una nueva pronunciación (fonología) y a un nuevo significado (semántica). La neurolingüística busca revelar estas operaciones mentales que nuestro cerebro realiza cuando aprende palabras o cualquier otro estímulo lingüístico en cada una de nuestras lenguas.

Gracias a las modernas técnicas de neuroimagen cerebral, sabemos que el cerebro es capaz de reconocer con extrema rapidez las letras y la pronunciación de una palabra, para luego recuperar su significado, tanto en la lengua materna (Holcomb y Grainger, 2006) como en la segunda lengua (Kroll, Gullifer, y Rossi, 2013). Por ejemplo, se sabe que nuestro cerebro necesita tan sólo 100 milisegundos para distinguir entre dos fonemas o sonidos dentro de una palabra, como distinguir entre /da/ y /ba/ (Näätänen, 2001). Más impresionante aún, cuando nuestro cerebro reconoce una palabra (por ejemplo, casa) bastan 400 milisegundos para que active de manera simultánea el significado de otras palabras similares en cuanto a su ortografía (como masa), su pronunciación o fonología (por ejemplo, caza) y su significado (hogar). Si pensamos que el cerebro bilingüe es capaz de activar simultáneamente no sólo las palabras que son similares dentro de una lengua, sino también aquellas que pertenecen a la otra lengua, entendemos por qué nos es tan difícil no pensar en el significado de diversión cuando vemos la palabra diversion en inglés.

\section{Aprender nuevas palabras}

El aprendizaje de vocabulario en una lengua extranjera implica establecer la asociación de nuevas unidades ortográficas (letras) y fonológicas (pronunciación) a un significado ya establecido en nuestra propia lengua. El aprendizaje de cada una de las nuevas palabras provocará cambios en las redes neuronales de la corteza cerebral que sustentan el conocimiento léxico. Gracias al registro de la actividad eléctrica del cerebro, por medio de la técnica de potenciales relacionados con eventos (PRES), es posible determinar de qué forma el cerebro bilingüe adquiere e integra nuevas palabras a su repertorio léxico y en qué medida el reconocimiento de las palabras, en cada una de las lenguas, comparte los mismos procesos mentales en el cerebro. LOS PRES nos permiten monitorear los cambios que se generan en la actividad eléctrica del cerebro de los estudiantes, incluso desde las primeras horas de aprendizaje de una lengua extranjera. 
Figura 1. Participante realizando un estudio electrofisiológico mientras lee palabras en el Laboratorio de lingüística en la Facultad de Lenguas y Letras de la Universidad Autónoma de Querétaro. Lleva puesto un gorro con electrodos conectados a un amplificador de la actividad eléctrica del cerebro.

Fuente.Autoría propia.
El registro de los PRES se obtiene mediante el uso de una serie de electrodos colocados en la piel cabelluda de los participantes (ver figura 1). Estos electrodos son capaces de detectar cualquier fluctuación de voltaje que resulta de la actividad neuronal en la corteza cerebral. Estas fluctuaciones de voltaje, generadas por la comunicación que se establece entre diferentes grupos de neuronas, se asocian a procesos mentales durante el reconocimiento de una palabra. Así, cualquier cambio en la actividad eléctrica del cerebro puede revelar la activación del significado de una palabra en una u otra lengua (ver figura 2). Cabe señalar que esta técnica ha sido ampliamente productiva e informativa en estudios neurolingüísticos sobre el acceso léxico en monolingües y bilingües, dadas sus características no invasivas, su bajo costo y su capacidad para ser utilizada en distintas poblaciones de niños y adultos sin ningún riesgo asociado. ${ }^{1}$

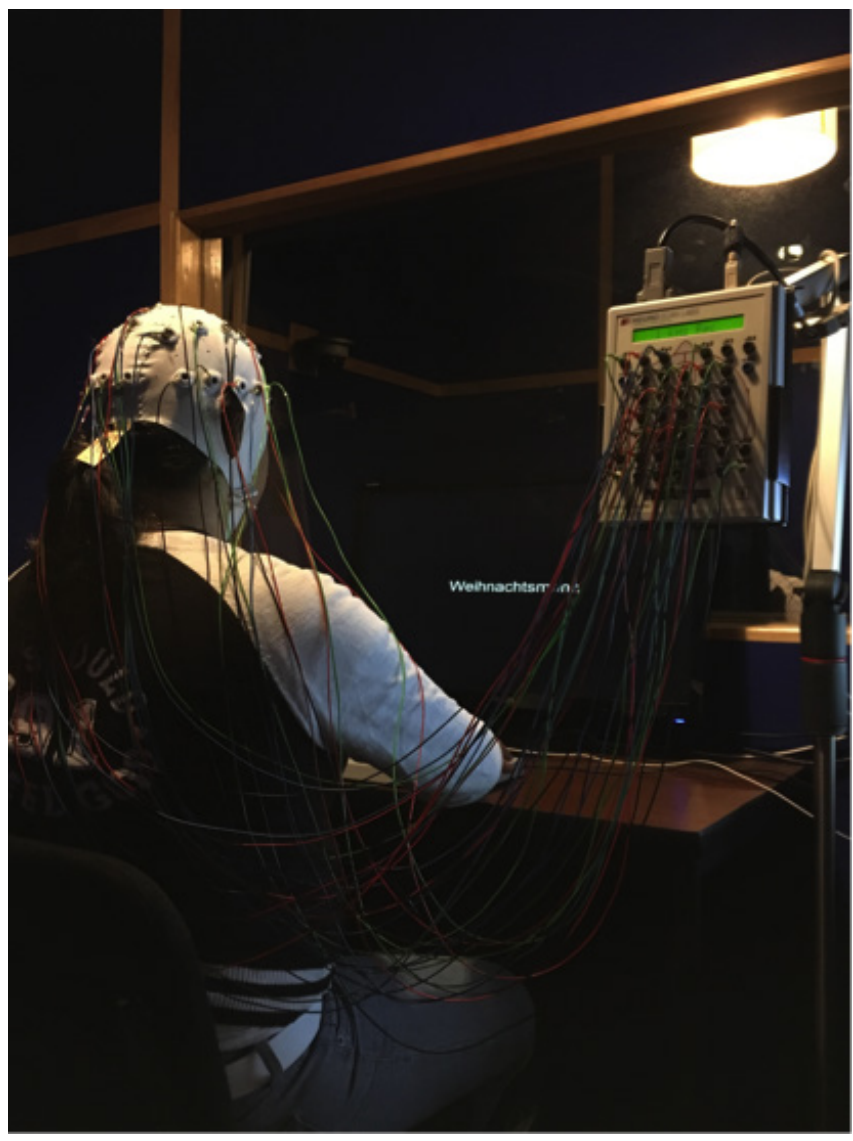

A partir del estudio con alumnos universitarios que aprendían una lengua extranjera en un contexto escolar, McLaughlin y sus colaboradores (2004) demostraron que, con tan sólo 14 horas de clase, el cerebro era capaz de reconocer si una palabra pertenecía o no al repertorio léxico de la lengua aprendida. ${ }^{2}$ Dichos cambios en la respuesta cerebral ocurrieron incluso a pesar de que los estudiantes no fueron capaces de identificar de manera explícita, mediante el uso de un cuestionario, entre las palabras reales o falsas de la lengua recién aprendida. Este tipo de estudios electrofisiológicos son importantes para el ámbito educativo porque demuestran que el cerebro adulto es capaz de aprender mucho más de lo que en realidad es observable a partir de las evaluaciones escolares tradicionales.

Asimismo, en una serie de estudios (Mulik, Carrasco-Ortíz y Amengual, 2018; Mulik, Pimentel y Carrasco-Ortiz, 2019) realizados en nuestro laboratorio encontramos que, en tan sólo tres sesiones de aprendizaje, los estudiantes universitarios mexicanos pueden aprender con mayor facilidad las palabras en una lengua extranjera, como el eslovaco, cuando la pronunciación se parece a otras palabras

1 Para saber más sobre el uso de esta técnica y la neurolingüística ver https://www.youtube.com/ watch?V=xA2EMO $420 \mathrm{C}$.

2 Para ver ejemplos de este tipo de estudios se puede consultar la siguiente página http:// vocabulary.ugent.be/wordtest/ del español. Por ejemplo, la palabra kura que en eslovaco significa "pollo" y se pronuncia como la palabra cura en español, fue mucho más fácil de aprender en comparación con otras palabras del eslovaco que no contienen ningún tipo de similitud con el español. Pareciera como si el hecho de contar con sonidos o fonemas similares en nuestra lengua materna nos ayudara a integrar estas nuevas palabras a nuestro cerebro bilingüe. 
Figura 2. Animación que muestra los cambios (en microvolts +-4) de la actividad eléctrica del cerebro de un grupo de bilingües mientras leen palabras en inglés entre 300 y 500 milisegundos después de haber visto las palabras. Fuente: adaptado de Carrasco-Ortiz et al., 2012
Además, la respuesta cerebral asociada al reconocimiento de estas palabras eslovacas recientemente aprendidas confirma la hipótesis de que el cerebro hace uso de los códigos fonológicos o sonidos que ya existen en nuestra lengua materna y los asocia con un nuevo significado en la lengua recién aprendida. Este tipo de evidencia nos podría ayudar a identificar aquellas palabras que, por ejemplo, para una persona con problemas de lenguaje o dislexia requerirían de un menor esfuerzo para aprender en clase (Nergis, 2011).
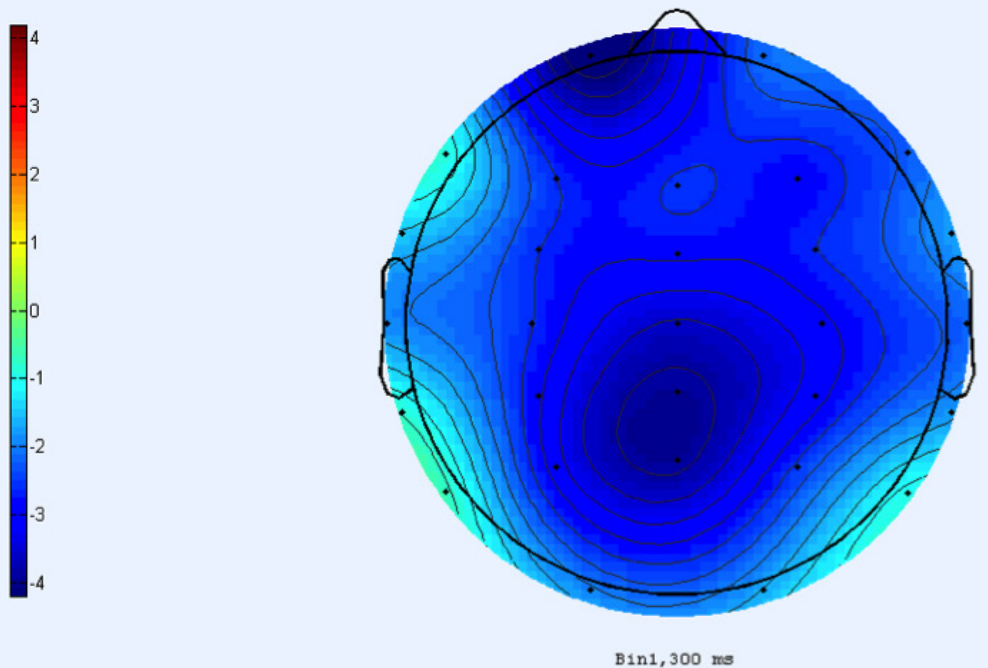

\section{Interacción entre palabras}

Cuando nos encontramos con una palabra en inglés como sale (que en español quiere decir "en venta") es muy difícil no pensar en una palabra conocida en español como salir. Si se tratara de un nativo hablante del francés, éste muy probablemente pensaría en la palabra sale, que en francés significa "sucio". Activar las palabras de nuestra lengua cuando leemos o escuchamos una palabra en otra lengua pareciera ser muy común y nuestro cerebro lo hace de una manera extremadamente rápida, que apenas nos damos cuenta de ello. Si hemos alcanzado una alta competencia en la otra lengua, podría incluso venir a nuestra mente el significado de una palabra en esa lengua cuando estamos hablando o leyendo en nuestra lengua materna. Es decir, podríamos pensar en una gran oferta cuando vemos la palabra sale mientras leemos en español.

De esta manera, todas las palabras de todas las lenguas que conocemos estarían interactuando entre sí en nuestro lexicón mental, con el fin de mantenerlas disponibles en caso de necesitarlas. Efectivamente, una de las hipótesis con mayor evidencia empírica en el área del bilingüismo es la que postula el acceso no selectivo del lenguaje (Dijkstra y van Heuven, 2002). Esto quiere decir que todas las unidades ortográficas, fonológicas y semánticas que forman las palabras estarían almacenadas en nuestro cerebro y que estas unidades nos servirían para formar todas las palabras que conocemos en todas 
"Neurolingüística: de cómo el cerebro bilingüe aprende palabras"

Figura 3. Respuesta neuronal en un electrodo central (Cz) de un grupo de nativos del francés que leían palabras en inglés. La flecha muestra una mayor negatividad en la respuesta eléctrica del cerebro de los participantes entre 300 y 600 milisegundos (componente N400, ver Kutas y Federmeier, 2011) después de haber leído las palabras con alta similitud fonológica entre francés e inglés (línea continua) en comparación con las palabras con baja similitud fonológica entre las dos lenguas (línea punteada). Fuente: adaptado de Carrasco-Ortiz et al., 2012 las lenguas que hemos aprendido. Esto significaría que la activación de una palabra en una lengua activaría de manera parcial y simultánea otra palabra de un idioma alternativo.

Lo anterior fue comprobado mediante un estudio electrofisiológico (Carrasco-Ortiz, Midgley y Frenck-Mestre, 2012), donde se buscó determinar en qué medida un grupo de bilingües podía activar las palabras de su L1 (francés) durante la lectura de palabras en su L2 (inglés). Por ejemplo, la palabra nid 'nido' en francés se pronuncia de manera muy similar a la palabra knee 'rodilla' en inglés. Los resultados de los bilingües mostraron una respuesta cerebral diferente entre las palabras con alta y baja similitud fonológica con las de su L1 (ver figura 3). Estos hallazgos sugieren que el cerebro de los bilingües activaría también las palabras de su lengua materna que tuvieran una pronunciación similar a las palabras que se presentaron en inglés.

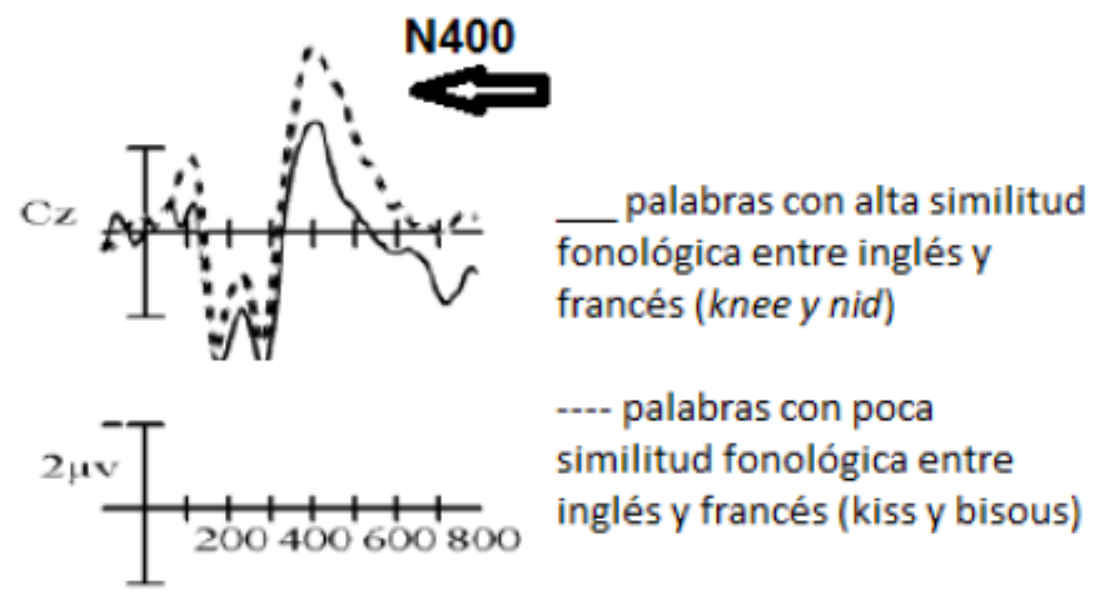

Más sorprendente aún, es poder observar el fenómeno inverso, es decir que el cerebro bilingüe active palabras de su segunda lengua mientras está leyendo palabras de su lengua materna. Esto ha sido observado en adultos bilingües que tienen una alta competencia en su L2 (Midgley, Holcomb y Grainger, 2011; van Hell y Dijkstra, 2002), pero también se ha encontrado el mismo fenómeno desde las primeras etapas del aprendizaje en estudiantes universitarios (Meade, Midgley, Dijkstra y Holcomb, 2018). Estos estudios ponen en evidencia el hecho de que las palabras de todas las lenguas que hemos aprendido conviven e interactúan entre sí y que varias de ellas pueden responder al llamado de activación si la palabra que se escucha o se lee tiene un alto grado de similitud con las palabras que conocemos, incluso antes de saber de qué lengua se trata. Sin embargo, si nuestro cerebro no tuviera un mecanismo para inhibir o "apagar" aquellas palabras que no corresponden completamente a la palabra buscada, no seríamos capaces de mantener una conversación o de comprender un texto en una u otra lengua. Aun así, pareciera más redituable y eficaz para el cerebro activar un mayor número de palabras potencialmente correctas con el fin de facilitar su reconocimiento. 
"Neurolingüística: de cómo el cerebro bilingüe aprende palabras"

Haydee Carrasco Ortiz y Flora Beatriz Hernández Carrillo

\section{Conclusiones e implicaciones para la enseñanza y el aprendizaje de lenguas}

Los recientes resultados de estudios neurolingüísticos nos han permitido poner en evidencia cambios importantes en la estructura funcional del cerebro después de la instrucción formal de una lengua extranjera. Cabe resaltar en estos estudios la capacidad del cerebro adulto para aprender e integrar nuevas palabras a su repertorio léxico en tan sólo unas cuantas horas de aprendizaje. La rapidez con la que el cerebro adulto integra nueva información léxica a su sistema lingüístico lo pone incluso en ventaja con respecto a los niños que aprenden una L2 (Gaskell y Ellis, 2009). Ésta puede deberse a que los adultos utilizan el repertorio léxico de su lengua materna, no únicamente para inferir el significado de las nuevas palabras, sino también para aprender su forma ortográfica y pronunciación. Esto quiere decir que conforme vamos aprendiendo nuevas palabras, vamos estableciendo vínculos o redes con las formas ortográficas y fonológicas presentes en nuestra lengua. De esta forma, el proceso de aprendizaje se verá beneficiado para aquellas nuevas palabras que tengan un patrón ortográfico similar al de las palabras de nuestra lengua. Asimismo, las palabras de la nueva lengua que puedan pronunciarse utilizando los mismos sonidos o fonemas presentes en nuestra lengua serán mucho más fáciles de aprender y de reconocer. ${ }^{3}$

Los hallazgos de los estudios neurolingüísticos interesados en entender los procesos de aprendizaje de una L2 pueden tener una repercusión importante, particularmente en el aprendizaje y evaluación del vocabulario. Esto coincide con lo que señala Cendoya (2009) respecto a que en el aula se puede trabajar con el conocimiento lingüístico previo de los estudiantes y no sólo con los encontrados en el entorno escolar de la L2. Igualmente, los estudios neurolingüísticos intentan disminuir la brecha que existe entre el ámbito de las neurociencias cognitivas y la práctica docente de las lenguas, como lo señaló la Organización para la Cooperación y el Desarrollo en 2007 para vincular las ciencias del aprendizaje y del cerebro (Cendoya, 2009). En un futuro no muy lejano, los estudios neurolingüísticos podrían proveernos de mayor conocimiento sobre el impacto de los entornos educativos y las prácticas pedagógicas en la estructura funcional del cerebro de los aprendientes de una lengua, ya sea en el aprendizaje de léxico o de estructuras gramaticales.

Finalmente, gracias a los estudios neurofisiológicos se ha podido demostrar

${ }^{3}$ En esta liga se recomiendan algunos apoyos audiovisuales para mejorar y evaluar el aprendizaje del vocabulario en inglés: https://learnenglish. britishcouncil.org/vocabulary y https://www.oxfordonlineenglish. com/english-level-test/ vocabulary. que el cerebro bilingüe es capaz de integrar información léxica desde las primeras horas de su aprendizaje, incluso a un nivel superior al que puede ser observado de manera conductual (por ejemplo, con la aplicación de un examen). El monitoreo de la actividad neuronal de los estudiantes desde las primeras horas de su aprendizaje aportará información relevante sobre los mecanismos cognitivos involucrados en el aprendizaje de nuevas palabras. Esta información ofrecerá indicadores adicionales para realizar ajustes en la intervención educativa y las formas de evaluar los aprendizajes en caso de ser necesario. 


\section{Referencias}

* Carrasco-Ortiz, H., Midgley, K. J., y Frenck-Mestre, C. (2012). Are phonological representations in bilinguals language specific? An ERP study on interlingual homophones. Psychophysiology, 49(4), 531-543. https://doi.org/10.1111/j.14698986.2011.01333.x

* Cendoya, A. (2009). Bilingüismo y Cerebro: Implicaciones para la Educación. Psicología educativa, 15(1), 39-44.

Dijkstra, T., y Van Heuven, W. J. B. (2002). The architecture of the bilingual word recognition system: From identification to decision. Bilingualism: Language and Cognition, 5(3), 175-197.

- Gaskell MG, Ellis AW. (2009). Word learning and lexical development across the lifespan. Philosophical transactions of the Royal Society of London. Series B, Biological sciences, 364, 3607-3615. https://doi.org/10.1098/rstb.2009.0213.

* Holcomb, P. J., y Grainger, J. (2006). On the time course of visual word recognition: an event-related potential investigation using masked repetition priming. Journal of Cognitive Neuroscience, 18, 1631-1643.

* Kroll, J. F., Gullifer, J. W., y Rossi, E. (2013). The Multilingual Lexicon: The Cognitive and Neural Basis of Lexical Comprehension and Production in Two or More Languages. Annual Review of Applied Linguistics, 33, 102-127. https://doi.org/10.1017/ S0267190513000111.

* Kutas, M., \& Federmeier, K. D. (2011). Thirty years and counting: Finding meaning in the $[400 \mathrm{~N}]$ component of the event-related brain potential (ERP). Annual Review of Psychology, 62, 14.1-14.27.

* Näätänen, R. (2001). The perception of speech sounds by the human brain as reflected by the Mismatch Negativity (MMN) and its magnetic equivalent (mmnm) Psychophysiology, 38,1-21.

* McLaughlin, J., Osterhout, L., y Kim, A. (2004) Neural correlates of second-language word learning: minimal instruction produces rapid change. Nature Neuroscience, 7,(7), 703-704.

* Meade, G., Midgley, K. J., Dijkstra, T., and Holcomb, P. J. (2018). Cross-language neighborhood effects in learners indicative of an integrated lexicon. Journal of Cognitive Neuroscience, 30, 70-85.

* Midgley, K. J., Holcomb, P. J., y Grainger, J. (2011). Effects of Cognate Status on Word Comprehension in Second Language Learners: An ERP Investigation. Journal of Cognitive Neuroscience, 23(7), 1634-1647. https://doi.org/10.1162/jocn.2010.21463.

* Mulík, S., Carrasco-Ortiz, H., y Amengual, M. (2018). Phonological activation of first language (Spanish) and second language (English) when learning third language (Slovak) novel words. International Journal of Bilingualism, 23(5), 1024-1040. https:// doi.org/10.1177/1367006918781061. 
* Mulík, S., Pimentel, M. y Carrasco-Ortiz, H. (2019). L1 and L2 phonological activation in L3 lexical learning: an ERP study [póster en la 12th International Symposium on Bilingualism].

* Nergis, A. (2011). To what extent does neurolinguistics embody EFL teaching methods? Procedia Social and Behavioral Sciences, 15, 143-147.

* Rastelli, S. (2018). Neurolinguistics and second language teaching: A view from the crossroads. Second Language Research, 34 (1), 103-123.

* Van Hell, J. G., y Dijkstra, T. (2002). Foreign language knowledge can influence native language performance in exclusively native contexts. Psychonomic Bulletin \& Review, 9(4), 780-789.

Wong, B., Yin, B. y O’Brien, B. (2016). Neurolinguistics: Structure, Function, and Connectivity in the Bilingual Brain. BioMedical Research International, 2016, 1-24.

\section{Cómo citar este artículo}

* Carrasco Ortiz, Haydee y Hernández Carrillo, Flora B. (2020). Neurolingüística: de cómo el cerebro bilingüe aprende palabras. Revista Digital Universitaria (RDU) Vol. 21, núm. 3 mayo-junio. Dol: http://doi.org/10.22201/codeic.16076079e.2020. v21n3.a6.

Recepción: 22/10/2019. Aceptación: 07/02/2020. 